\title{
Generation of high drug loading amorphous solid dispersions by Spray Drying
}

\section{Costa, B. L. A.*; Sauceau, M. ; Sescousse, R.; Ré, M. I.}

Université de Toulouse; Ecole des Mines d’Albi; UMR CNRS 5302; Centre RAPSODEE, F-81013 Albi, France

*E-mail of the corresponding author: bhianca.lins_de_azevedo_costa@mines-albi.fr

\begin{abstract}
Amorphous solid dispersions (ASDs) refers to drug - carrier systems, where the drug is dispersed in the carrier at a molecular level. In this work, ASDs were formulated by spray drying. The aim was to achieve high drug loads of an amorphous hydrophobic drug (Efavirenz-EFV) in the carrier Soluplus ${ }^{\circledR}$. Solid state characterizations (mDSC, XRPD, DVS, Raman, SEM, stability and solubility studies) were done. EFV amorphisation in ASDs (20 to 85\% EFV loads) resulted in improved drug solubility compared to unprocessed EFV crystals and tendency of properties evolution over time for ASDs with EFV loads higher than $70 w t \%$.
\end{abstract}

Keywords: amorphous solid dispersion, spray drying, high drug loading, soluplus, efavirenz. 


\section{Introduction}

Poorly water-soluble drugs have steadily grown on the global pharmaceutical industry. The technological approach focused on rendering the drug amorphous to improve apparent solubility remains a challenge since amorphous state is metastable in nature with a potential to undergo recrystallization [1]. In order to prevent this conversion, amorphous materials have been stabilized as solid dispersions using generally hydrophilic carriers for stabilization [2].

An amorphous solid dispersion (ASD) refers to drug-carrier systems in which the mechanism of drug dispersion is the key to understand its behavior. Such formulations impart an antiplasticizing effect on the amorphous compound yielding an increase in the glass transition temperature thereby reducing molecular mobility [3]. However, in order to achieve adequate stabilization, solid dispersions are often produced with a relatively low drug load ( $<30 \mathrm{wt} \%)$ dispersed in the carrier at molecular level. The problem is that a low drug-loaded ASD requires a large dose to ensure therapeutic efficacy.

The insertion of high drug load formulations on the market is expected to meet patients demand for fixed, unique and smaller dosage combinations products. Furthermore, the supersaturated combinations may reduce dosage amounts as well as decrease the production in the pharmaceutical industries to supply cost savings. The aim of the current work was to generate, by spray drying, ASDs comprising a hydrophobic drug class II BCS (low solubility, good permeability for oral administration) in a hydrophilic carrier (Soluplus ${ }^{\circledR}-\mathrm{SOL}$ ) expecting to achieve drug loads higher than $40 \% \mathrm{w} / \mathrm{w}$ in the binary mixtures. The physical stability will be the critical control point. The drug of choice is Efavirenz (EFV). EFV is a non-nucleoside reverse transcriptase inhibitor used in the first-line treatment of HIV with low solubility (3$9 \mu \mathrm{g} / \mathrm{mL}$ ) and high permeability [4].

\section{Materials and Methods}

EFV was kindly supplied by Cristalia Ltd (Itapira, Brazil), SOL (polyvinyl caprolactampolyvynil acetate-polyethylene glycol) was obtained from BASF corporation (Ludwigshafen, Germany) and Ethanol (Carlo Erba, Italy) was used as organic solvent.

The feeding solution was prepared by dissolving EFV in a $10 \mathrm{wt} \%$ solution of Soluplus in ethanol. Binary mixtures EFV-SOL containing from 20 to $85 \mathrm{wt} \%$ of EFV were formulated as spray-dried powders. The individual constituents (drug and polymer) were also spray-dried from ethanol solutions and used for comparison purposes.

A Buchi B-290 minispray dryer (Buchi Labortechnik AG, Flawil, Switzerland) equipped with Inert Loop B-295 and an integrated two-fluid $0.7 \mathrm{~mm}$ nozzle was used to produce the ASD samples. Compressed nitrogen was used as the drying/carrying gas with a flow rate of $600 \mathrm{~L} / \mathrm{h}$. The solution feed rate was typically $3 \mathrm{~g} / \mathrm{min}$, the inlet temperature was set to $80 \pm 2{ }^{\circ} \mathrm{C}$ and the outlet temperature was maintained at $59 \pm 2{ }^{\circ} \mathrm{C}$. 
X-ray diffraction (XRD), modulated differential scanning calorimetry (mDSC), Raman spectroscopy, Scanning electron microscopy (SEM) and Infrared Spectroscopy (IR) were used to characterize the solid state of the spray-dried samples.

Water sorption isotherms were determined gravimetrically using an automated dynamic water sorption analyzer (DVS). Samples were subjected to 0-95 \% relative humidity (RH) sorptiondesorption cycle, over $10 \%$ RH increments. For stability studies, samples were analyzed using DVS and exposed to stress conditions at $40{ }^{\circ} \mathrm{C}$ and $75 \%$ RH during 15 days. Every 3 days, the samples were quickly analyzed by DSC to monitor changes in the physical state.

Solubility studies of unprocessed EFV crystals and ASDs were carried out in duplicate. For that purpose, an excess amount of samples was added into $30 \mathrm{~mL}$ of a dissolution medium containing purified water plus $0.25 \mathrm{wt} \%$ of sodium lauryl sulfate (SLS) under agitation at $37 \pm 0.5^{\circ} \mathrm{C}$ in a water bath. The withdraws were collected from 5 min until $48 \mathrm{~h}$.

\section{Results and discussion}

\subsection{X-ray diffraction (XRD)}

The absence of Bragg peaks in X-ray diffractograms (Fig. 1) of all spray-dried solids indicates the complete loss of the crystalline structure of EFV, which becomes amorphous during the spray-drying process.

\subsection{Thermal analysis (mDSC)}

Thermal analysis was performed to investigate the apparent EFV-Soluplus ${ }^{\circledR}$ miscibility. Fig. 2 shows the single experimental glass transition temperature $\left(\mathrm{T}^{\mathrm{g}}\right)$ identified for pure contituents and for each binary mixture EFV-SOL loaded with 40, 60 and $85 \mathrm{wt} \% \mathrm{EFV}$. The $T^{g}$ of mixtures $\left(\mathrm{T}^{\mathrm{g}}{ }_{\text {mix }}\right)$ are placed as an intermediary between the pure drug and polymer values and are close to the theoretical values of $T^{g}$ calculated by Gordon-Taylor (GT) equation (Equation 1). This equation relates the individual contributions of each component in an ideal mixture (with no interactions between the components).

$$
T_{m i x}^{g}=\frac{W_{P A} \cdot T_{P A}^{g}+K \cdot\left(1-W_{P A}\right) \cdot T_{P O}^{g}}{W_{P A}+K \cdot\left(1-W_{P A}\right)}
$$

$\boldsymbol{T}_{\boldsymbol{P A}}^{\boldsymbol{g}}$ and $\boldsymbol{T}_{\boldsymbol{P O}}^{\boldsymbol{g}}$ are the drug and polymer glass transition temperatures (K), respectively. $\boldsymbol{W}_{\boldsymbol{P A}}$ is the drug mass fraction in the mixture. $\mathrm{K}$ is defined as fitting parameter characterizing the curvature of the evolution and is defined by equation 2 [5].

$$
K=\frac{T_{P A}^{g} \cdot \rho_{P A}}{T_{P O}^{g} \cdot \rho_{P O}}
$$

Densities $\left(\mathrm{g} / \mathrm{cm}^{3}\right)$, determined by helium picnometry, $\boldsymbol{\rho}_{\boldsymbol{P A}}$ and $\boldsymbol{\rho}_{\boldsymbol{P} \boldsymbol{O}}$, are 1.39 and 1.18, respectively. 
According to XRD and DSC results, an amorphous solid dispersions of EFV- SOL seems to be formed during the spray-drying process.

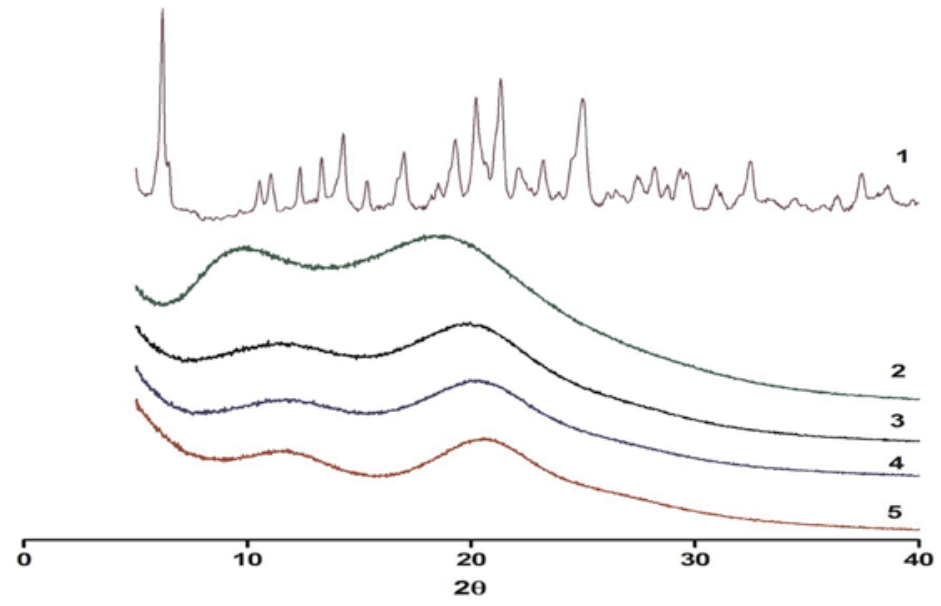

Fig. 1 XRD diffractograms of: (1) pure EFV; (2) pure SOL; (3) $40 \%$ EFV; (4) $60 \%$ EFV and (5) $85 \%$ EFV.

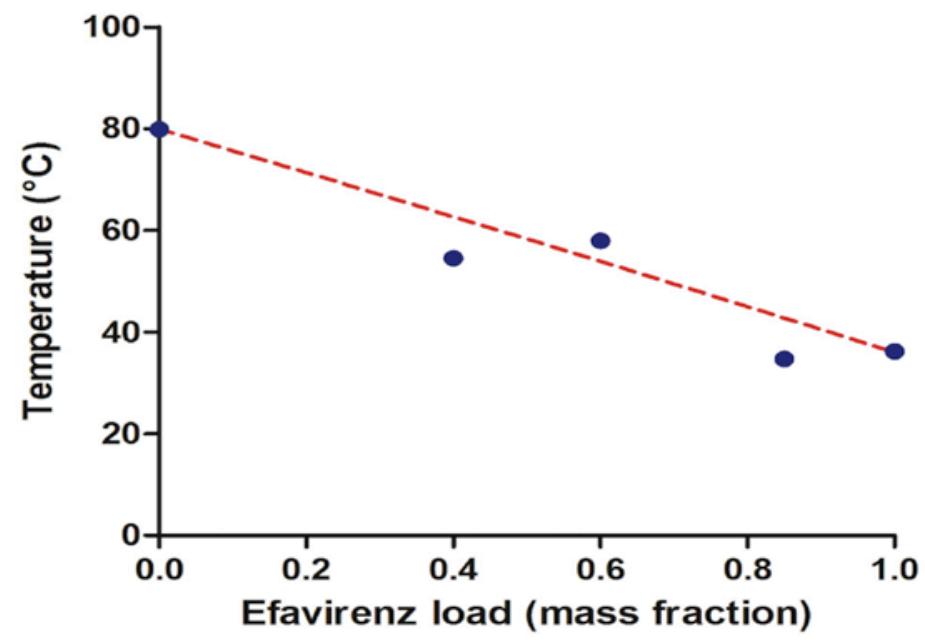

Fig. 2 Glass transition temperature ( $T^{g_{\text {mix }}}$ ): measured (blue circles) and theoretical values by GT equation (dotted red line).

\subsection{Raman microscopy}

Raman microscopy was also performed for three EFV-SOL ASD (Fig. 3). They were evaluated by observing the characteristic peaks of pure drug (peak at $2250 \mathrm{~cm}^{-1}$ ) and pure polymer (peak at $2900 \mathrm{~cm}^{-1}$ ). As expected, the increase of the drug load in the mixture corresponded to a more 
intense characteristic EFV peak. The presence of characteristic peaks of pure components in all ASD Raman spectra shows the absence of specific interactions between the two components and confirms the ideal mixing suggested by the evolution of the mixtures $T^{g}$ and SOL for all studied drug loads.

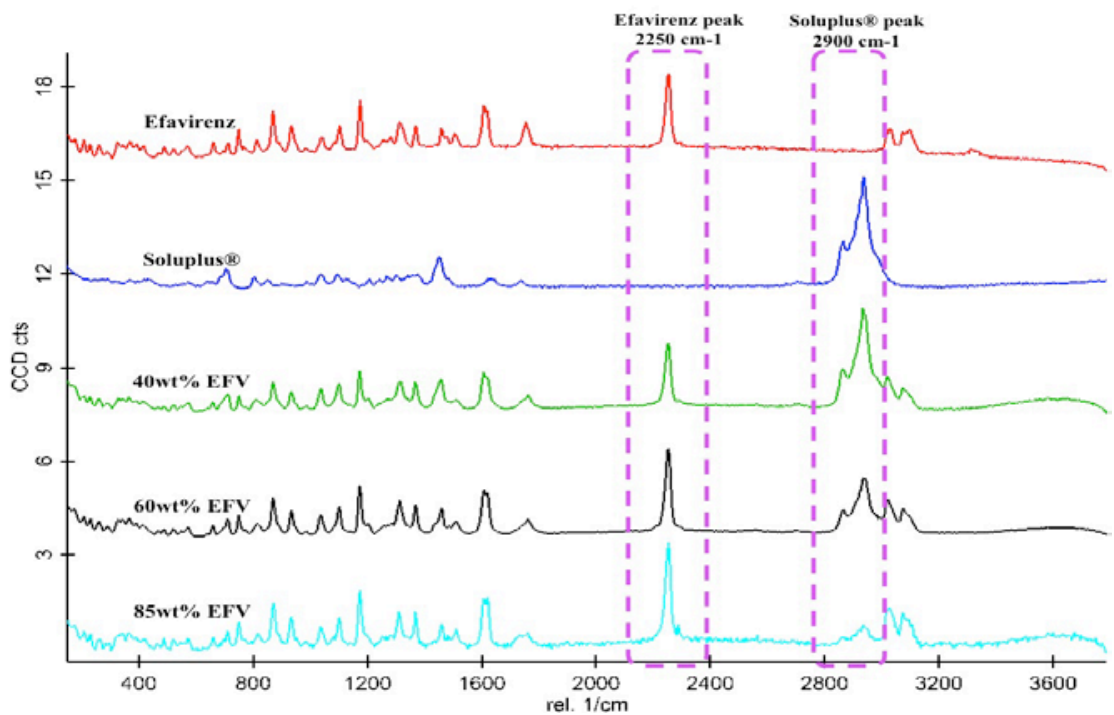

Fig. 3 Raman spectra for the EFV-SOL ASD with different drug loads: 40\%; 60\%; 85\% EFV.

\subsection{Scanning electron microscopy (SEM)}

SEM images of the spray-dried powders are shown in Fig. 4. ASD EFV-SOL particles are predominantly spherical and different in shape from both original constituants that are the unprocessed EFV crystals (Fig. 4B - long rods with regular and organized multi-face geometry) and the spray-dried polymer (Fig. 4A - wrinkled particles). A good mixing between both compounds could led to these apparently homogeneous solid particles.

\subsection{Stability studies}

Fig. 5 displays the DVS isotherm plots for the studied samples, showing the change in mass percentage as a function of changing relative humidity. The reversibility of the water uptake was clearly seen in all cases. Taking as example the curves at $75 \%$ RH (Fig. 5B), the tendency of increasing the hydrophobic character of EFV-SOL ASD by increasing the EFV load is demonstrated. The decreased affinity to water with increased drug load could be an interesting attribute for the physical stability of the amorphous drug phase in high drug load ASDs. 


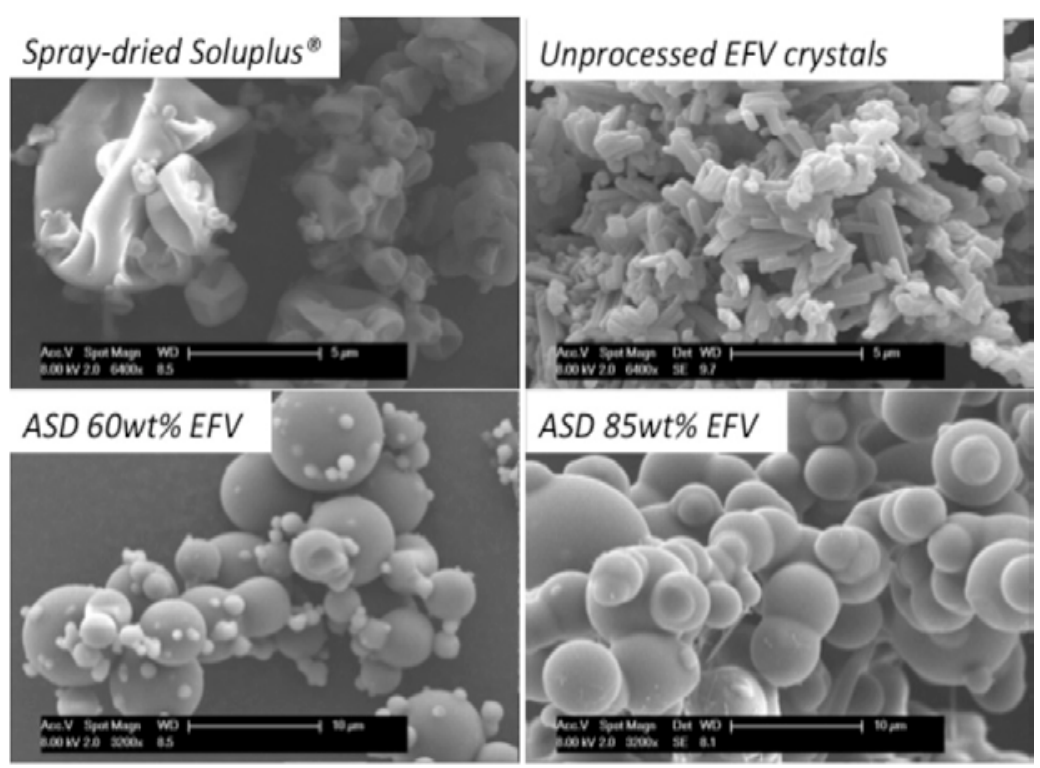

Fig. 4 SEM images of spray-dried ASD constituted by a EFV-SOL binary mixture.

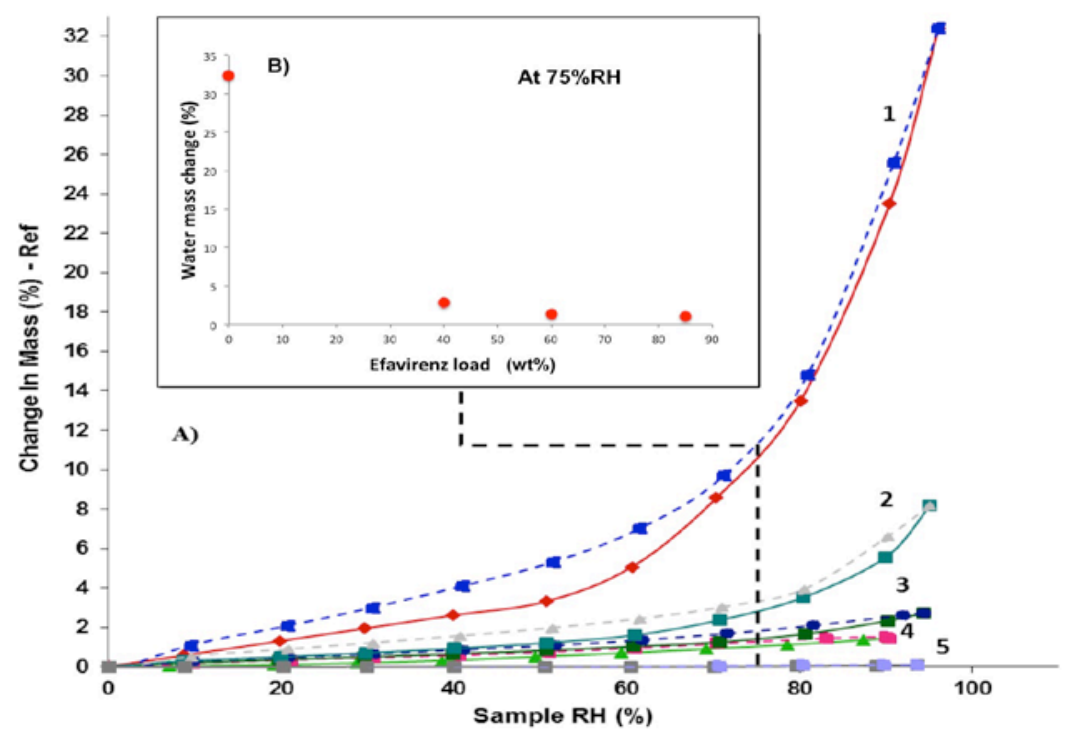

Fig. 5 A) DVS isotherms for 1- pure SOL; 2- 40\% EFV; 3- 60\% EFV; 4- 85\% EFV; 5- pure EFV. B) Water mass change (\%) at $75 \% \mathrm{RH}$ for the ASD.

The stability results under stress conditions are presented in Table 1 . It is noticeable that the samples have evolved over time since $\mathrm{T}^{\mathrm{g}}$ mix increases in both cases, suggesting a variation in the amorphous mixture composition. However, we did not observe any particular signs of 
amorphous phase separation or drug recrystallisation for the sample loaded with $40 \% \mathrm{EFV}$. Contrarily, 70 \% EFV-SOL ASD revealed an evolution of crystallization enthalpy $(\Delta \mathrm{H})$ over time, corresponding to a partial EFV recrystallization. This tendance for recrystallization can be attributed to the high drug load of the ASD, probably corresponding to a supersaturated (and instable) amorphous state of the drug. Considering $\Delta \mathrm{H}=52.83 \mathrm{~J} / \mathrm{g}$ for the unprocessed EFV crystals, it was possible to estimate the percentage of drug recrystallizing in this ASD during storage. Table 1 also shows that, after 15 days of storage, approximately $6.6 \%$ of the amorphous EFV dispersed in Soluplus recrystallized.

\subsection{Solubility studies}

In Figure 6, the results showed that spray-dried EFV-SOL ASDs produced with 20, 40 and 60 $w t \%$ EFV presented a good solubility enhancement in comparison with the unprocessed EFV crystals. EFV-SOL ASDs solubility decreases gradually with drug load increasing in the spraydried powders. Indeed, EFV- SOL ASD with 20wt\% EFV exhibited the maximum apparent EFV solubility over approximately $36 \mathrm{~h}$ in simulated intestinal fluid.

Table 1. Stability results under stress conditions $\left(40{ }^{\circ} \mathrm{C} / 75 \% \mathrm{RH}\right)$ for EFV-SOL ASD with drug loads of $40 \%$ and $70 \%$

\begin{tabular}{|c|c|c|c|c|}
\hline \multirow[b]{2}{*}{ t (days) } & \multirow{2}{*}{$\begin{array}{c}40 \% \text { EFV- } \\
\text { SOL } \\
T_{\text {mix }}^{g}\left({ }^{\circ} C\right)\end{array}$} & \multicolumn{3}{|c|}{$70 \%$ EFV-SOL } \\
\hline & & \multicolumn{2}{|c|}{$\begin{array}{c}\text { Crystallizatior } \\
\text { enthalpy } \\
\Delta \mathrm{H}(\mathrm{J} / \mathrm{g})\end{array}$} & $\begin{array}{l}\text { Crystallization } \\
\text { (\%) }\end{array}$ \\
\hline 3 & 59.8 & 51.4 & 1.3 & 2.4 \\
\hline 6 & 64.9 & 53.4 & 2.6 & 4.9 \\
\hline 9 & 64.1 & 55.9 & 2.8 & 5.3 \\
\hline 15 & 67.2 & 57.7 & 3.5 & 6.6 \\
\hline
\end{tabular}

\section{Conclusions}

A robust formulation with optimal drug load and excipients is one of the key factors of successfully developing an ASD system. In this work, amorphous solid dispersions of the poorly water soluble compound Efavirenz were prepared with drug loads in the range 20 to $85 \mathrm{wt} \%$ EFV, using Soluplus ${ }^{\circledR}$ as hydrophilic carrier and spray drying as the production process. Solubility tests revealed a good solubility enhancement for all ASD compared with crystalline drug solubility. To the best of our knowledge, it is the first study reporting such high levels of drug loading (up to 85 wt\%) in supersaturated amorphous solid dispersions of 
EFV. However, our results also showed that the physical stability should be a monitoring critical point for EFV-SOL ASDs with EFV loads higher thanv $70 \mathrm{wt} \% \mathrm{EFV}$.

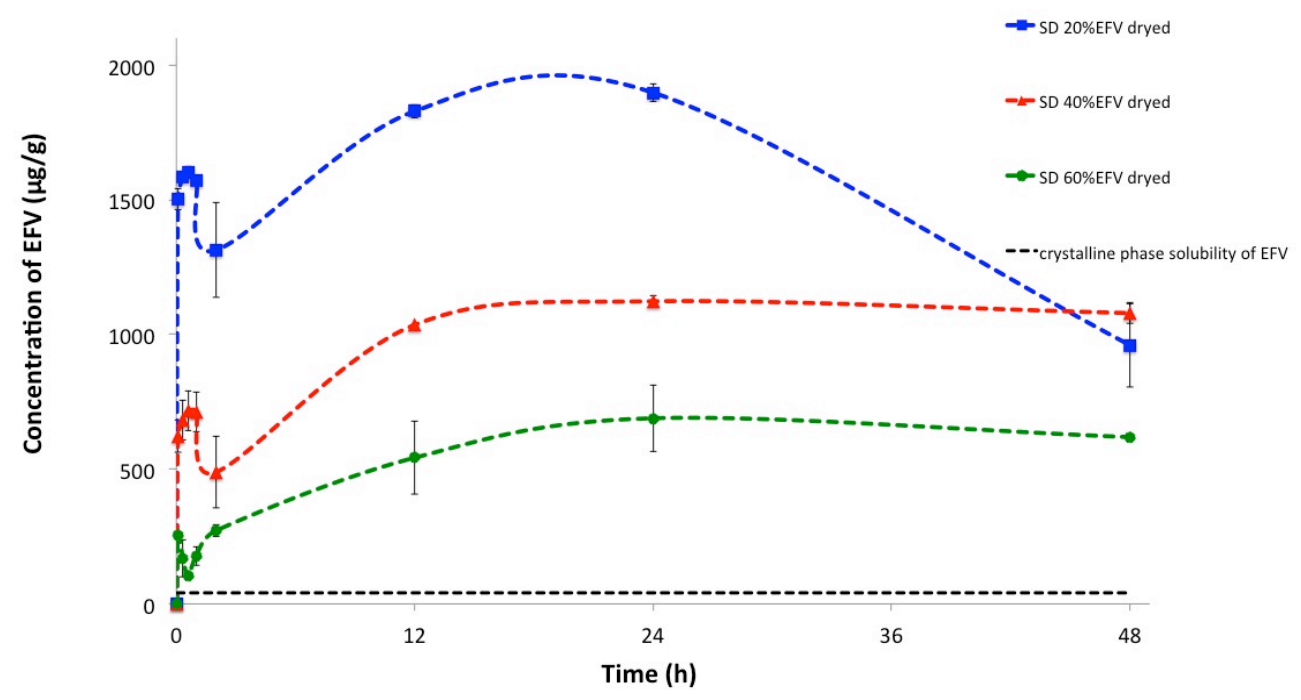

Fig. 6 Kinetic solubility of spray-dried EFV-SOL ASD.

\section{References}

[1] K. Wlodarski, W. Sawicki, A. Kozyra, and L. Tajber, 'Physical stability of solid dispersions with respect to thermodynamic solubility of tadalafil in PVP-VA', Eur. J. Pharm. Biopharm. Off. J. Arbeitsgemeinschaft Pharm. Verfahrenstechnik EV, vol. 96, pp. 237-246, Oct. 2015.

[2] Z. Lu et al., 'Supersaturated controlled release matrix using amorphous dispersions of glipizide’, Int. J. Pharm., vol. 511, no. 2, pp. 957-968, Sep. 2016.

[3] B. B. Patel, J. K. Patel, S. Chakraborty, and D. Shukla, 'Revealing facts behind spray dried solid dispersion technology used for solubility enhancement’, Saudi Pharm. J., vol. 23, no. 4, pp. 352-365, Sep. 2015.

[4] C. R. D. Hoffmeister et al., 'Efavirenz dissolution enhancement III: Colloid milling, pharmacokinetics and electronic tongue evaluation', Eur. J. Pharm. Sci. Off. J. Eur. Fed. Pharm. Sci., vol. 99, pp. 310-317, Mar. 2017.

[5] J. A. Baird and L. S. Taylor, 'Evaluation of amorphous solid dispersion properties using thermal analysis techniques’, Adv. Drug Deliv. Rev., vol. 64, no. 5, pp. 396-421, Apr. 2012. 